\title{
Unity of Opposite: Highly Emissive Luminogens in both Solution and Aggregate States toward Room Temperature Phosphorescence and Electroluminescence
}

\author{
Pengbo Han, ${ }^{1}$ Chengwei Lin, ${ }^{1}$ Yejun Yao, ${ }^{1}$ Jia Wang, ${ }^{1}$ Yanping Qiu, ${ }^{1}$ Dongge Ma, ${ }^{1, *}$ Anjun Qin ${ }^{1, *}$ and \\ Ben Zhong Tang1,2,3
}

${ }^{1}$ State Key Laboratory of Luminescent Materials and Devices, Guangdong Provincial Key Laboratory of Luminescence from Molecular Aggregates, Center for Aggregation-Induced Emission, South China University of Technology, Guangzhou, 510640, China. E-mail: msdgma@scut.edu.cn (D.G. Ma); msqinaj@scut.edu.cn (A.J. Qin)

${ }^{2}$ Shenzhen Institute of Molecular Aggregate Science and Engineering, School of Science and Engineering, The Chinese University of Hong Kong, Shenzhen, 2001 Longxiang Boulevard, Longgang District, Shenzhen City, Guangdong 518172, China.

3 Hong Kong Branch of Chinese National Engineering Research Center for Tissue Restoration and Reconstruction, The Hong Kong University of Science and Technology, Clear Water Bay, Kowloon, Hong Kong, China.

\begin{abstract}
Organic light-emitting materials, especially those with two-phase high emission, have attracted considerable attention for applications in bioimaging agents, sensors, optoelectronic devices, etc. Many fluorophores applied in such fields either emit brightly in dilute solution or in aggregate state, with the former often suffering from aggregation-caused quenching effect, and the latter falling dark at low concentrations. Herein, we overcame the dilemma by balancing the planar and distorted structures with various side units and achieved bright emission in both dilute solution (e.g., the absolute quantum yields $\left(\Phi_{\mathrm{PL}}\right)=90.2 \%$ in THF) and in aggregate states (e.g., $\Phi_{\mathrm{PL}}=92.7 \%$ in powder state, $\Phi_{\mathrm{PL}}=95.3 \%$ in crystal). These luminescent materials are demonstrated as promising guests embedded into host matrix to achieve efficient room temperature phosphorescence, and these host-guest systems could be applied in the information encryption. Moreover, these luminogens could also be used as single-component emitting layers to construct non-doped organic light-emitting diodes, from which a maximum external quantum efficiency up to $4.75 \%$ with Commission International de L'Eclairge (CIE) coordinates of (0.15, 0.05), which is neatest to next generation ultra-high definition television (UHDTV) display standard, was realized. This work provides a feasible strategy of balancing the planar and distorted structure of a luminogen toward highly efficient emission in both solution and solid states.
\end{abstract}

\section{INTRODUCTION}

Organic luminescent materials are the promising candidates as organic light-emitting diodes (OLEDs), ${ }^{1-3}$ bioimaging agents, ${ }^{4-5}$ sensors, memory devices ${ }^{6-8}$, etc. The fluorophores with simple molecular structure, facile tunability and thermal stability are desirable for the construction of excellent molecular devices. ${ }^{9-12}$ However, traditional fluorophores often suffer from the aggregation-caused quenching (ACQ) effect, in which they emit intensely in dilute solution while exhibit weak or even no emission in aggregate state. ${ }^{13-15}$ In contrast, the luminogens with aggregation-induced emission feature (AIEgens) are weakly or non-emissive in dilute solution while emit brightly in aggregate state. ${ }^{16-20}$ Although these two phenomena are converse, and their luminescence principle are relative each other, it is meaningful and challenging to construct the fluorophores that emit strongly in both their solution state and aggregate state.

Scientists have paid much efforts toward this goal. For examples, Stang et al. reported two tetragonal prismatic metallacages by using self-assembly chemistry to achieve intense emission in both single molecule and aggregate state. ${ }^{21}$ Yang et al. developed the self-assembled purely organic stack to produce bright fluorescence in two-phase states, which in turn overcomes the putative disadvantage of heavy metals complexes. ${ }^{22}$ However, the specific stereogeometry should be required for these self-assembly stacks. Besides the self-assembly chemistry, several molecular structural engineering was also performed for the construction of such two-phase highly emissive molecules. For instance, Yuan, Zhang and Tang et al. designed two triphenylamine derivatives with intense emission in isolate state (the quantum yield $\left(\Phi_{\mathrm{PL}}\right)=79.4 \%$ in DMF) and aggregate state ( $\Phi_{\mathrm{PL}}=54.8 \%$ in crystal) via the conjugation-induced rigidity effect. ${ }^{23}$ Zhu and Zhao et al. prepared fluorophores by combing a planar diphenyldiacetylene core with distorted cyanostilbene unit, which emit intensely with $\Phi_{P L}$ of $98.2 \%$ in THF, but the emission became weaker with $\Phi_{\mathrm{PL}}$ decreasing to $60.7 \%$ in solid state. ${ }^{24}$ Subsequently, Zhu et al. achieved a two-phase balanced fluorophore with thermally activated delayed fluorescence feature via a rigid-and-flexible alternation design approach. Obviously, the $\Phi_{\mathrm{PL}}$ values are both 


\section{(A)}


(D)

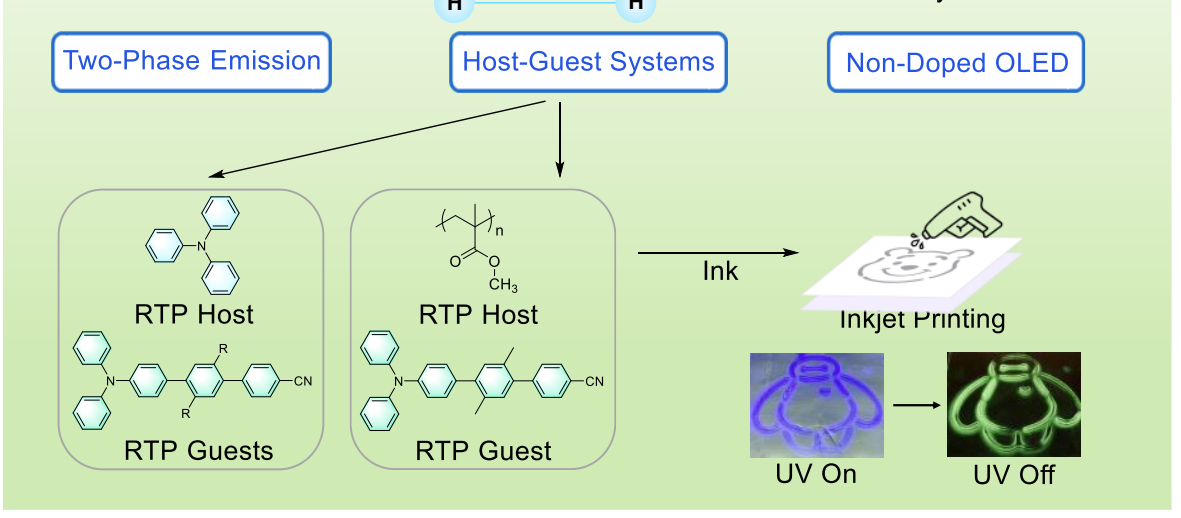

Figure 1. Schematic illustration of combined structural engineering for the multifunctional two-phase high fluorescent materials. (A) Molecular design strategy for two-phase highly emissive fluorophores. (B) Two-phase high emission characteristic of the fluorophores. (C) The strategy used to develop efficient RTP materials with RTP host and RTP guest to construct a doping system, and these host-guest systems are applied in the information display and encryption. (D) The non-doped OLEDs fabricated by utilizing the high emissive fluorophores as emitting layers.

lower than $35.3 \% .^{25}$ Therefore, it is quite difficult to simultaneously realize highly emissive fluorophores with $\Phi_{\mathrm{PL}}$ values both higher than $90 \%$ in solution and solid state, and a simple and more accurate molecular design strategy is highly desired.

Inspired by these elegant works, herein, a simple and rational molecular design strategy was proposed to obtain the luminogens with two-phase high emission. In consideration that substantial rigidity is needed to limit intramolecular motions ${ }^{26-27}$, a planar terphenyl, a moiety with ACQ effect was selected as the core to construct highly emissive molecules in solution state (Figure 1A). ${ }^{28}$ The terphenyl core with planar conformation can greatly limit intramolecular rotation and maintain the maximal electronic conjugation. On the other hand, since brightly emissive molecule in solid state requires considerable twisting conformation to prevent the excimer formation, a twisted triphenylamine (TPA) was used to suppress intermolecular $\pi-\pi$ stacking. ${ }^{29-31}$ Moreover, cyano $(\mathrm{CN})$ unit was also attached to terphenyl to achieve the donor-acceptor (D-A) molecules. The D-A fluorophores might also be planar in dilute solution due to the change of the bond length and conformation relax of the excited-state molecule, further enhancing their emission in solution state. ${ }^{24}$ Moreover, the intermolecular $\mathrm{C}-\mathrm{H} \cdots \mathrm{N}$ hydrogen bonds in adjacent molecules can efficiently inhibit molecular rotation and improve molecular horizontal orientation, ${ }^{32-33}$ which in turn improve their emission property in solid state. Furthermore, the side chains were added to the terphenyl core with the anticipation of altering molecular conformation to fine-turn their emission.

Therefore, three terphenyl derivatives of 2PB-AC, 2Me2PB-AC and 2T2PB-AC with the same D and A decorated by different side chains were designed and synthesized. Unlike the traditional ACQ fluorphores (ACQphores) and AIEgens, 2PB-AC, 2Me2PB-AC and 2T2PB-AC show strong fluorescence in their powder and crystalline states, as well as their solution states with absolute quantum yields higher than $90 \%$ (Figure 1B). Benefiting from their high emission efficiency in dilute solution, 2PB-AC, 2Me2PB-AC and 2T2PB-AC were demonstrated as the promising guests embedded into crystalline host and polymethyl methacrylate (PMMA) matrix to achieve intense emission in such solid solution (Figure 1C). Furthermore, these doped systems also exhibit efficient room temperature phosphorescence (RTP), and can be applied in the information encryption, which provides a new platform to the development of purely organic RTP materials. Moreover, thanks to their superior thermal stability and high emission efficiency in their solid states, three non-doped OLEDs were fabricated by utilizing 2PB-AC, 2Me2PB-AC and 2T2PB-AC as single-component emitting layers (EMLs) (Figure 1D). Among them, the 2Me2PB-AC-based OLED exhibits a maximum external quantum efficiency (EQE) of $4.75 \%$ with Commission International de L'Eclairge (CIE) coordinates of $(0.15,0.05)$, 
which is amenable to next generation ultra-high definition television (UHDTV) display standard. ${ }^{34,35}$ The strategy used in this work represents a concise but more precise approach for the construction of such highly emissive luminogens in both solution and aggregate/solid states, which might further facilitate their practical applications.


Figure 2. Emission behaviors in solution state. (A) UV-vis and PL spectra of 2PB-AC, 2Me2PB-AC and 2T2PB-AC in THF solution. (B) PL peak and absolute quantum yields of 2PB-AC, $2 \mathrm{Me} 2 \mathrm{~PB}-\mathrm{AC}$ and 2T2PB-AC in solution. $\lambda_{\mathrm{ex}}: 330 \mathrm{~nm}$. Concentration: $10 \mu \mathrm{M}$. CNNPI is a traditional TICT molecule. Emission behavior in powder state. (C) PL spectra and (D) lifetime decay profiles of the emission band at 468, 415 and $437 \mathrm{~nm}$ of 2PB-AC, 2Me2PB-AC and 2T2PB-AC powders, respectively. $\lambda_{\text {ex }}: 330 \mathrm{~nm}$.

\section{RESULTS AND DISCUSSION}

After confirming their structures spectroscopically, the photophysical properties of 2PB-AC, 2Me2PB-AC and 2T2PB-AC were investigated in solution state. As shown in Figure 2A, the absorption bands located at 300-400 nm originate from the intramolecular charge transfer (ICT) transition. ${ }^{36}$ Notably, the absorption peak of $2 \mathrm{MePB}-\mathrm{AC}$ and 2T2PB-AC are slightly blue-shifted compared with that of 2PB-AC, suggesting that their $\pi$-conjugation are weakened via the introduction of substituents in $2 \mathrm{~PB}-\mathrm{AC}$. Upon excitation, 2PB-AC, 2MePB-AC and 2T2PB-AC emit in blue and sky-blue region with peaks at 475, 460 and $483 \mathrm{~nm}$ in THF solution, respectively (Figure $2 \mathrm{~A}$ ). In comparison with $2 \mathrm{~PB}$ $\mathrm{AC}\left(\lambda_{\mathrm{em}}=475 \mathrm{~nm}\right)$, the photoluminescence $(\mathrm{PL})$ peak of $2 \mathrm{Me} 2 \mathrm{~PB}-\mathrm{AC}$ gives a $15 \mathrm{~nm}$ blue-shift, whereas a red-shift of $8 \mathrm{~nm}$ is observed in the PL peak of 2T2PB-AC, which might be ascribed to the weaker $\pi$-conjugation and the extension of charge transfer (CT) component, respectively. It is worth noting that these compounds possess large Stokes shift $(\Delta \lambda)$ of $118 \sim 179 \mathrm{~nm}$. Moreover, as demonstrated in Figure 2B and S1, 2PB-AC, 2MePB-AC and 2T2PB-AC show distinct solvatochromism effect with bright emission in different solvents. For instance, 2PB-AC presents strong emission with PL quantum yields $\left(\Phi_{\mathrm{F}}\right)$ higher than $83 \%$ in different solvents. The $\Phi_{\mathrm{F}}$ of $2 \mathrm{~PB}-\mathrm{AC}, 2 \mathrm{MePB}-\mathrm{AC}$ and $2 \mathrm{~T} 2 \mathrm{~PB}-\mathrm{AC}$ in THF were recorded to be $90.2 \%, 80.1 \%$ and $67.8 \%$, respectively. Interestingly, their $\Phi_{\mathrm{F}}$ gradually increases along with red-shifted emission peak, which is different from the fluorophores with twisted intramolecular transfer (TICT) feature, ${ }^{37-38}$ such as CNNMI. ${ }^{39}$ These different photophysical behaviors imply that these compounds emit from planar intramolecular charge transfer (PICT) excited state instead of a TICT state. ${ }^{40}$

To further understand their intense emission in dilute solution, the computational study was performed by using time-dependent density functional theory (TD-DFT). The optimized ground- and excited-state conformation, as well as the lowest unoccupied molecular orbital (LUMO) and the highest occupied molecular orbital (HOMO) of 2PB-AC, 2Me2PB-AC and 2T2PB-AC, were plotted in Figure S2. Their HOMOs are mainly located on the triphenylamine moiety, with a slight delocalization onto the adjacent benzene ring, while their LUMOs are mainly concentrated on the terphenyl and cyano groups (Figure S2A). The spatial separation of HOMO and LUMO could result in an effective ICT transition, which is also confirmed by their distinct solvatochromism effect. The molecular structure in the excited state exhibits a more planar conformation than in ground state, evidenced through the reduction of torsion angles (Figure S2B). The planar excited-state conformation can effectively suppress the molecular motions and open the radiative decay channel, and thus a bright emission was observed in dilute solution. Moreover, the $\Phi_{\mathrm{F}}$ of $2 \mathrm{Me} 2 \mathrm{~PB}-\mathrm{AC}$ decreases slightly compared with that of $2 \mathrm{~PB}-\mathrm{AC}$ in dilute solution, which is attributed to a more twisted molecular conformation due to the introduction of methyl-substituents. Furthermore, the introduction of sulfur atom can facilitate efficient intersystem crossing (ISC) process, which in turn further reduces the $\Phi_{\mathrm{F}}$ of $2 \mathrm{~T} 2 \mathrm{~PB}-\mathrm{AC}$ in dilute solution. ${ }^{41,42}$

Next, the photophysical properties of the powders of 2PBAC, 2MePB-AC and 2T2PB-AC were studied. As shown in Figure $2 \mathrm{C}$, they emit in violet and blue region with peaks at 468,415 , and $437 \mathrm{~nm}$, respectively. In contrast with isolated molecule in THF solution, the emission peaks of 2PB-AC, 2MePB-AC and 2T2PB-AC powder are considerably blueshifted, implying that a shorter conjugation length in solid state than that in solution. ${ }^{23}$ Notably, the $\Phi_{\mathrm{F}}$ values of $2 \mathrm{~PB}-$ $\mathrm{AC}, 2 \mathrm{Me} 2 \mathrm{~PB}-\mathrm{AC}$ and 2T2PB-AC powders are as high as $92.7 \%, 68.1 \%$ and $53.2 \%$, respectively. Above results indicate that these compounds can emit brightly in both solution and aggregate states, which integrate the advantages of AIEgens and ACQphores. Moreover, as depicted in Figure $2 \mathrm{D}$, the PL decay spectra of $2 \mathrm{~PB}-\mathrm{AC}, 2 \mathrm{MePB}-\mathrm{AC}$ and $2 \mathrm{~T} 2 \mathrm{~PB}-$ $A C$ present a single exponential decay process with ideally short exciton lifetimes $(\sim \mathrm{ns})$, which suggest they emit fluorescence. To further confirm the process, the fluorescent and phosphorescent spectra of 2PB-AC, 2MePB-AC and 2T2PB-AC powders were measured at $77 \mathrm{~K}$ to acquire experimental lowest excited single $\left(\mathrm{S}_{1}\right)$ and triplet $\left(\mathrm{T}_{1}\right)$ states (Figure S3). Their big experimental single-triplet energy splitting $\left(\Delta E_{\mathrm{ST}}\right)$ and short fluorescence lifetime might rule out the possibility of thermally activated delayed fluorescence. In addition, PL decay spectra of the emission band around $\mathrm{T}_{1}$ peak of $2 \mathrm{~PB}-\mathrm{AC}, 2 \mathrm{Me} 2 \mathrm{~PB}-\mathrm{AC}$ and $2 \mathrm{~T} 2 \mathrm{~PB}-\mathrm{AC}$ powders were also investigated. Their PL lifetimes were deduced to be to $7.67,3.63$, and $1.81 \mathrm{~ns}$, respectively (Figure 
(A)
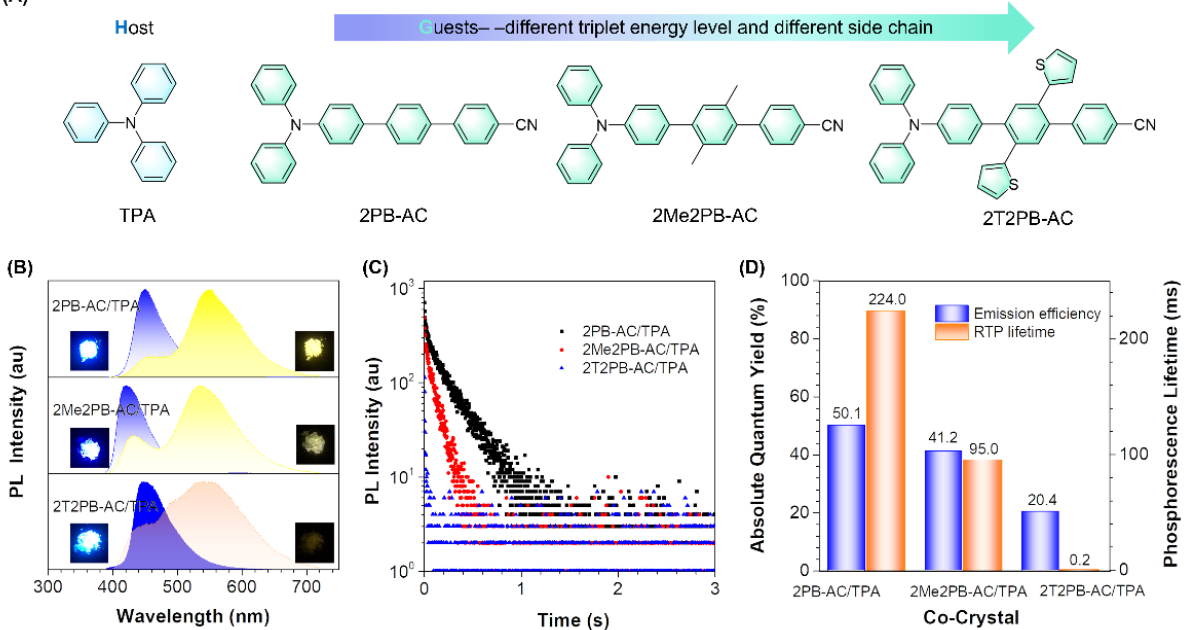

Figure 3. (A) The guest-host strategy for the development of efficient RTP materials from fluorescent hosts and guests. (B) Fluorescence (blue line) and phosphorescence (yellow line) spectra of host-guest crystalline materials. Inset: photographs of guest/TPA materials with and without UV irradiation. (C) Phosphorescence decay curves of host-guest crystalline materials. Weight ratio of guest and host $=1: 100 ; \lambda_{\text {ex: }} 330 \mathrm{~nm}$. (D) Emission efficiency and RTP lifetime of host-guest crystalline materials.

S4 and Table S1), further confirming that they are a group of fluorescent compounds.

To further decipher their solid-state emission mechanism, the single crystal of 2PB-AC (CCDC 2095218) was grown successfully from a DCM/n-hexane mixture by a slow solvent evaporation, and the crystal structure was analyzed by $X$-ray diffraction crystallography. The results show that 2PB-AC possesses a planar conformation with angles in the range of $5-46^{\circ}$ (Figure S5A). No close $\pi-\pi$ stacking was found, but multiple intermolecular $\mathrm{C}-\mathrm{H} \cdots \pi$ interactions with distances of 3.052-3.808 Å between the molecules existed (Figure S5B). Moreover, the strong intermolecular $\mathrm{C}-\mathrm{H} \cdots \mathrm{N}$ hydrogen bonds with a short distance of $2.645 \AA$ were also observed in adjacent molecules. The multiple and strong intermolecular interactions can effectively rigidify molecular conformation and reduce non-radiative energy dissipation in their aggregate states. ${ }^{35,43}$ Therefore, 2PB-AC crystal gives bright blue emission with a high $\Phi_{\mathrm{F}}$ of $95.3 \%$ (Figure S6). In addition, as depicted in Figure S7, the PL decay spectrum of 2PB-AC crystal exhibits a single exponential decay process with short exciton lifetimes ( $\sim \mathrm{ns})$, confirming that the 2PB-AC crystal also emits fluorescence.

Benefiting from their high emission efficiency in dilute solutions, 2PB-AC, 2MePB-AC and 2T2PB-AC can act as the guest embedded into crystalline host matrix with very low concentration to achieve bright emission in such solid solution. Meanwhile, the crystalline host matrix can effectively restrict the non-radiation transition of guest molecules, and in turn to achieve efficient room-temperature phosphorescence. ${ }^{44-48}$ Therefore, a facile strategy for realizing purely organic RTP was proposed by constructing the host-guest systems (Figure 3A). Wherein, TPA was used as a host according to previous works. ${ }^{45,49}$ The results show that all the host-guest systems present visible afterglow when the UV light is turned off (Figure 3B and Videos S1-S3). The $1 \mathrm{wt} \%$ 2PB-AC/TPA, 2Me2PB-AC/TPA and 2T2PB-AC/TPA blend systems emit deep blue light with peaks at 450, 422, $447 \mathrm{~nm}$, respectively (Table S2). Their $\Phi_{\mathrm{F}}$ values were also recorded to be $50.1 \%, 41.2 \%$ and $20.4 \%$, respectively. Both host and guests exhibit bright fluorescence, while the 2PB-AC/TPA, 2Me2PB-AC/TPA and 2T2PB-AC/TPA systems emit distinct phosphorescence with peaks at 548,534 and $560 \mathrm{~nm}$, respectively. The fluorescence and phosphorescence of $2 \mathrm{~PB}$ AC/TPA, 2Me2PB-AC and 2T2PB-AC/TPA systems both originate from the guests. The fluorescence lifetimes of $2 \mathrm{~PB}-$ AC/TPA (450 nm), 2Me2PB-AC/TPA (422 nm) and 2T2PBAC/TPA (447 nm) was deduced to be 3.04, 2.44 and $3.16 \mathrm{~ns}$, respectively (Figure S8). Whereas, the RTP lifetimes of $2 \mathrm{~PB}$ AC/TPA, 2Me2PB-AC/TPA and 2T2PB-AC/TPA systems were recorded to be to 224,95 , and $0.16 \mathrm{~ms}$ (Figure $3 \mathrm{C}$ and Figure 3D), respectively, resulting in the afterglow at different time scales observed with the naked eye.

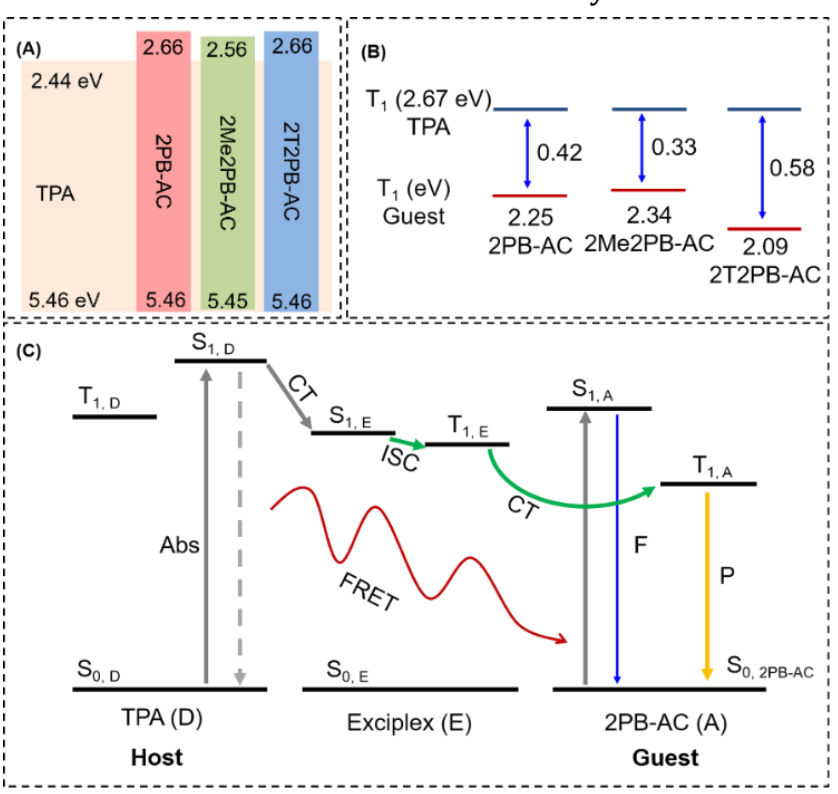

Figure 4. (A) HOMO and LUMO energy levels of host (TPA) and guests (2PB-AC, $2 \mathrm{Me} 2 \mathrm{~PB}-\mathrm{AC}$ and 2T2PB-AC). (B) The $\mathrm{T}_{1}$ energy levels of the TPA and guests. (C) Proposed energytransfer path between guests and host. 
(B)<smiles>COC(=O)C(C)(C)C(C)(C)C</smiles>

PMMA RTP Host

(C)

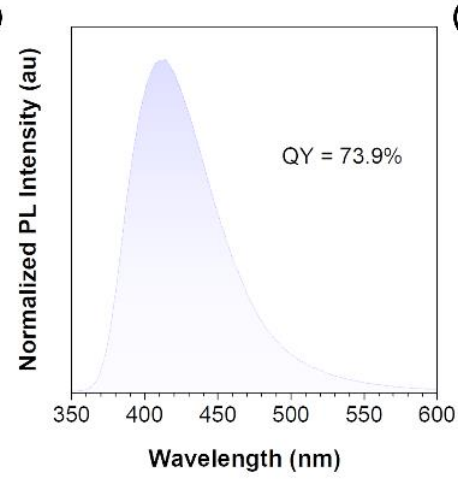

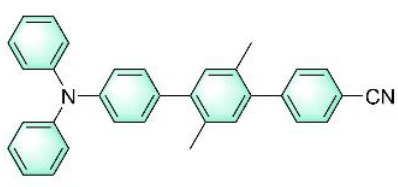

2Me2PB-AC

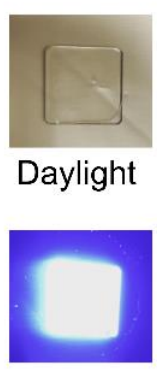

UV on $40 \mathrm{~s}$

(D)





(E)

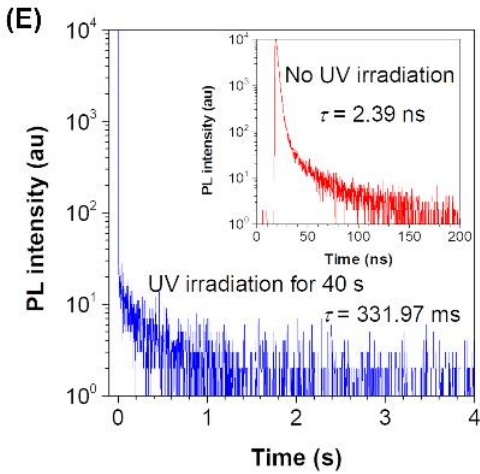

Figure 5. (A) The guest-host strategy for the development of efficient flexible RTP material from non-emissive host and fluorescent guest. (B) Photographs of the phosphors taken at different time intervals before and after removing the excitation of a 365 nm UV lamp. (C) Steady-state photoluminescence of 2Me2PB-AC@PMMA. (D) Phosphorescence spectra and (E) lifetime decay profiles of the emission band around $517 \mathrm{~nm}$ of 2Me2PB-AC@PMMA before and after photo-irradiation for $40 \mathrm{~s}$ under ambient conditions. $\lambda_{\text {ex: }} 330 \mathrm{~nm}$. Weight ratio of $2 \mathrm{Me} 2 \mathrm{~PB}-\mathrm{AC}$ and PMMA $=1: 100$. Samples were annealed at $90^{\circ} \mathrm{C}$ for 2 h.

To decipher emission mechanism of the host-guest systems, electronic properties of 2PB-AC, 2Me2PB-AC and 2T2PB-AC were measured to acquire the practical HOMO and LUMO energy levels by using cyclic voltammetry (CV). As shown in Figure S9, the HOMO values of $2 \mathrm{~PB}-\mathrm{AC}, 2 \mathrm{Me} 2 \mathrm{~PB}-\mathrm{AC}$ and 2T2PB-AC were estimated to be $-5.17,-4.96,-5.16 \mathrm{eV}$, respectively, whereas, their LUMO were deduced to be -2.66 , $-2.56,-2.66 \mathrm{eV}$, respectively, based on their oxidation and reduction onsets against $\mathrm{Fc} / \mathrm{Fc}^{+}$redox couple. The electronic property of TPA is also acquired from previous work. ${ }^{50}$ The HOMO and LUMO energy alignments of the host and guests were shown in Figure 4A. Moreover, $S_{1}$ and $T_{1}$ of host $\left(S_{1, D}\right.$ and $\left.T_{1, D}\right)$ and guests $\left(S_{1, A}\right.$ and $\left.T_{1, A}\right)$ were obtained from their emission peaks of fluorescence and phosphorescence spectra at $77 \mathrm{~K}$, respectively (Figures S3, S10 and S11). The energy gaps between $T_{1}$ of TPA and $T_{1}$ of guests were plotted in Figure 4B.

In addition, energy transfer and intermolecular interactions between host and guest are also crucial for the RTP behavior of the host-guest systems. Therefore, the Förster resonance energy transfer (FRET) processes between host and guest were evaluated. A great overlap between emission of the host and absorption of the guests is observed (Figure S12), which confirms the FRET process existed in such systems. During the transfer process, TPA acts as an energy donor and 2PB-AC or 2Me2PB-AC or 2T2PB-AC serves as an energy acceptor. $49,51-52$ Moreover, electron spin resonance (ESR) spectra of the host-guest systems exhibit a strong signal with $g$-factor of 2.002 at $298 \mathrm{~K}$ (Figure S13).
This value is close to that of a free electron (2.00232), indicative of the existence of unpaired electrons and radicals during CT process in the host-guest systems. ${ }^{53}$ Therefore, the intermediate CT state also plays a key role in the hostguest systems.

Based on these results, the proposed emission mechanism is depicted in Figure 4C. Taking 2PB-AC/TPA system as an example, singlet excited state is generated in TPA $\left(\mathrm{S}_{1, \mathrm{D}}\right)$ when the host-guest system is photoexcited. Since small HOMO and LUMO energy offset ( $\Delta E_{\text {номо-Luмо) of the TPA and }}$ 2PB-AC will facilitate fast intermolecular electron transfer, the generated excitons are converted into the exciplex singlet excited state $\left(\mathrm{S}_{1, \mathrm{E}}\right)^{44}$. Meanwhile, remaining excitons $\left(\mathrm{S}_{1, \mathrm{D}}\right)$ are fully converted into the singlet excited states $\left(\mathrm{S}_{1, \mathrm{~A}}\right)$ through the efficient FRET process between TPA and 2PBAC. Therefore, the fluorescence of TPA was not observed in PL spectra of the host-guest systems, and the generated singlet excited states $\left(S_{1, A}\right)$ emit strongly. Afterward, the generated exciplex singlet excited states $\left(\mathrm{S}_{1, \mathrm{E}}\right)$ effectively transform into the triplet excited states $\left(\mathrm{T}_{1, \mathrm{E}}\right)$ because of the small energy gap between them. The $\mathrm{T}_{1, \mathrm{E}}$ value should be slightly lower than $\mathrm{S}_{1, \mathrm{E}}$ and higher than $\mathrm{T}_{1, \mathrm{~A}}$ according to the $2 \mathrm{~PB}$ $\mathrm{AC} / \mathrm{TPA}$ fluorescence and $2 \mathrm{~PB}-\mathrm{AC}$ phosphorescence spectra. Therefore, $T_{1, E}$ could be quickly converted to $T_{1, A}$ via a CT process and gives an obvious phosphorescence..$^{44}$ However, the intermolecular CT process might gradually be reduced with the introduction of substituents. Moreover, the large $\mathrm{T}_{1}$ energy gaps between TPA and 2T2PB-AC are disadvantage 


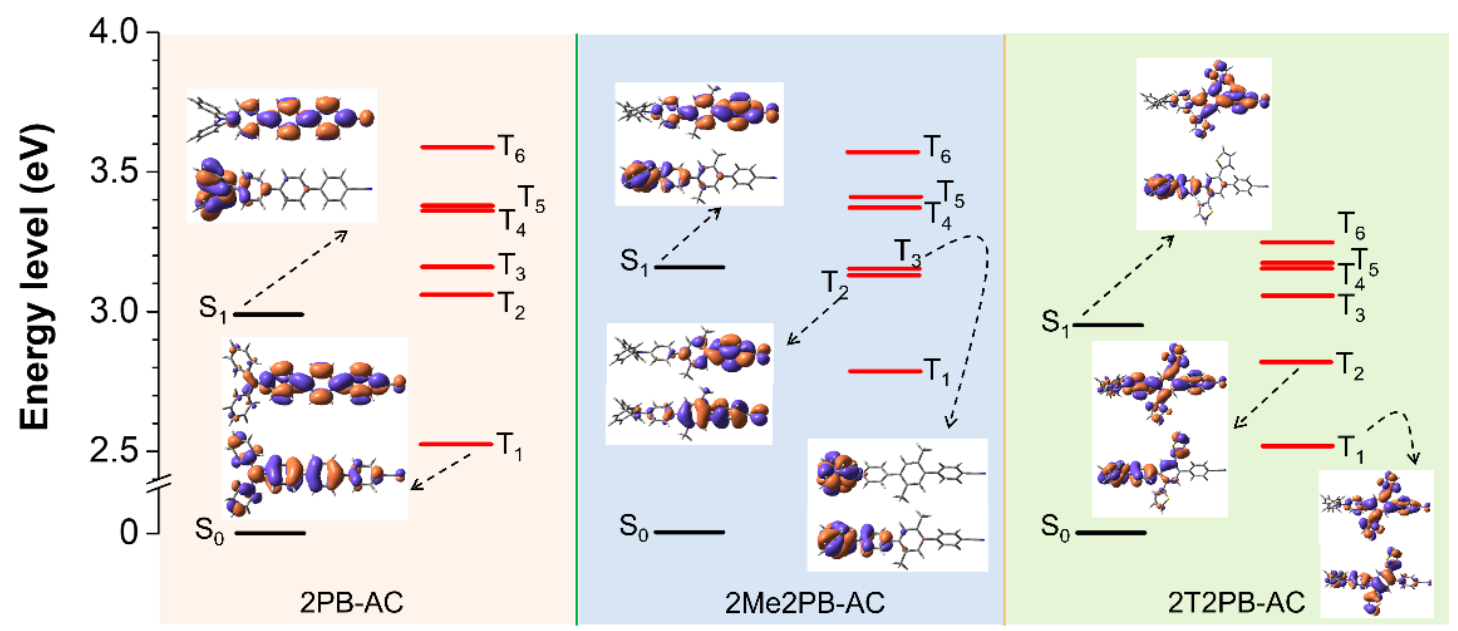

Figure 6. Relative excitation energies, their corresponding hole and electron wavefunctions of $2 \mathrm{~PB}-\mathrm{AC}, 2 \mathrm{Me} 2 \mathrm{~PB}-\mathrm{AC}$ and 2T2PB-AC (Hole and electron wavefunctions are shown below and above in the schematic, respectively).

for intermolecular CT process between $\mathrm{T}_{1, \mathrm{E}}$ and $\mathrm{T}_{1, \mathrm{~A}}$. Therefore, different RTP behaviors were found in the host-guest systems.

Besides using the crystalline host matrix, rigid PMMA was also adopted to stabilize the triplet excitons for the construction of efficient and flexible RTP systems (Figure 5A). After doped into PMMA matrix, only 2Me2PB-AC@PMMA shows notable RTP behavior. Notably, after continue photoirradiation using a 365 UV lamp for $40 \mathrm{~s}$, the phosphorescence of 2Me2PB-AC@PMMA could last for more than $5 \mathrm{~s}$ at room temperature (Figure $5 \mathrm{~B}$ and Video $\mathrm{S} 4$ ). As illustrated in Figure 5C, 2Me2PB-AC (1 wt\%)@PMMA exhibits a strong violet fluorescence with peak at $414 \mathrm{~nm}\left(\Phi_{\mathrm{F}}=73.9 \%\right)$. In its delay emission spectra (Figure $5 \mathrm{D}$, delay time $=0.1 \mathrm{~ms}$ ), no obvious phosphorescence was found. This is because the phosphorescence of $2 \mathrm{Me} 2 \mathrm{~PB}-\mathrm{AC}$ is almost quenched by $\mathrm{O}_{2}$ in PMMA matrix. ${ }^{54}$ However, after photo-irradiation for 40 $s$ under ambient condition and the same delay time, 2Me2PB-AC@PMMA exhibits a bright phosphorescence at $517 \mathrm{~nm}$ (Figure 5D and S14). Its PL lifetime of emission peak at $517 \mathrm{~nm}$ were recorded at $2.39 \mathrm{~ns}$ and $331.97 \mathrm{~ms}$ before and after photo-irradiation under ambient conditions, respectively (Figure 5E).

To analyze the extraordinary phosphorescence behavior, the 2Me2PB-AC@PMMA were placed in vacuum and air atmosphere, respectively. Only a strong green phosphorescence was observed by naked eyes under 365 UV light when it was placed in vacuum (Figure S15 and Video S5). In addition, 2Me2PB-AC also exhibits strong phosphorescence in THF solution at $77 \mathrm{~K}$ (Figure S16 and Video S6). Furthermore, phosphorescence property of 2Me2PB-AC was studied to further exclude the influence of molecule itself on the luminescence properties before and after photo-irradiation. The lifetimes of $2 \mathrm{Me} 2 \mathrm{~PB}-\mathrm{AC}$ powder with emission peak at 414 and $517 \mathrm{~nm}$ are almost no change before and after photo-irradiation for $40 \mathrm{~s}$ under ambient conditions (Figure S17), suggesting that the photo-activation phosphorescence of 2Me2PB-AC@PMMA is not ascribed to the change of molecule itself, such as crystal movement. ${ }^{55}$ Above results indicate that the rigid environment of PMMA matrix suppress the nonradiative decay of $2 \mathrm{Me} 2 \mathrm{~PB}-\mathrm{AC}$, thus making the doped system to show bright phosphorescence. Meanwhile, the photo-activated phosphorescence of 2Me2PB-AC @ PMMA might be attributed to the consumption of residual $\mathrm{O}_{2}$ in PMMA matrix, which is sensitized by $\mathrm{T}_{1}$ state of 2Me2PB-AC.

Why did only 2Me2PB-AC@PMMA present notable RTP behavior in these doped systems? As we well know, the fundamental requirement of the triplet emission is permitted to access the triplet excited state of the guests. Therefore, to have a deeper understanding of the ISC process of terphenyl derivatives, the excited state characteristics of 2PB-AC, 2Me2PB-AC and2T2PB-AC were investigated by TD-DFT calculations (Figure S18). The main triplet states were evaluated below the $S_{1}$ excited states because of thermodynamically favorable ISC route. ${ }^{56}$ As shown in Figure 6, in 2Me2PB-AC, two main ISC pathways were observed because of small $\Delta E_{\text {st }}$ and followed E1-Sayed rule by close-lying $\mathrm{S}_{1}-\mathrm{T}_{2}$ and $\mathrm{S}_{1}-\mathrm{T}_{3} .{ }^{57}$ However, for 2PB-AC and 2T2PB-AC, the close-lying triplet excited states exhibit similar molecular orbital (MO) conformation compared with $\mathrm{S}_{1}$, implying that the E1-Sayed rule is not permitted. In addition, there is a big $\Delta E_{\text {st }}$ between $\mathrm{S}_{1}$ and $\mathrm{T}_{1}$ of $2 \mathrm{~PB}-\mathrm{AC}$ and 2T2PB-AC. Therefore, no obvious RTP was observed for 2PB-AC @ PMMA and 2T2PB-AC@PMMA due to inhibited ISC process.

Based on the photo-activation RTP feature of the fabricated amorphous material, 2Me2PB-AC@PMMA can be used for information display and encryption through inkjet printing technology. As demonstrated in Figure 7A and 7B, eight patterns including different cartoon animals and flowers were fabricated by using 2Me2PB-AC@PMMA as ink, and then these patterns were dried at $90{ }^{\circ} \mathrm{C}$ for $2 \mathrm{~h}$. These patterns show bright and clear green phosphorescence after photo-irradiation for $40 \mathrm{~s}$ under ambient conditions (Videos S7-S14). Such flexible and highly efficient RTP materials will be perfectly suitable for diverse applications, such as anti-counterfeiting, inkjet printing and information storage without complicated fabrication process.

On the other hand, 2PB-AC, 2Me2PB-AC and 2T2PB-AC can also act as single-component EMLs to construct nondoped OLEDs because of their high emission efficiency in aggregate states. To select appropriate device fabrication 


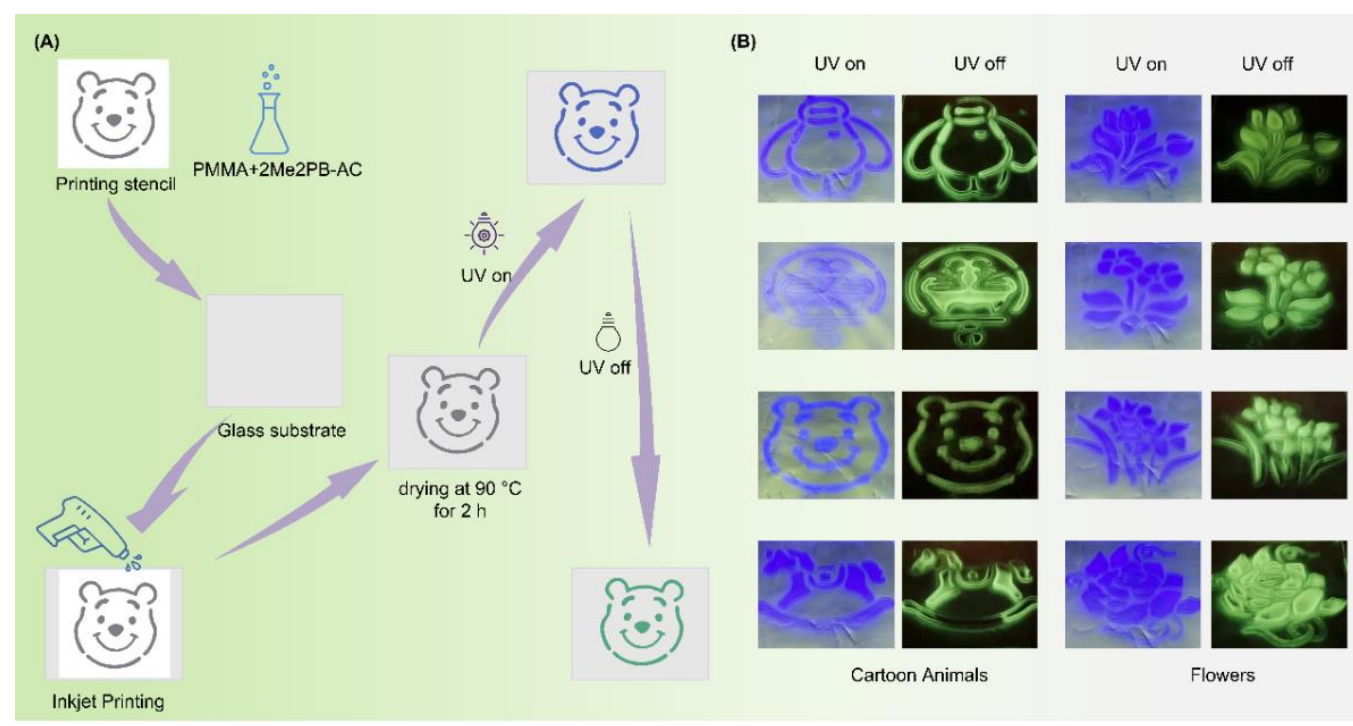

Figure 7. (A) Inkjet printing patterning. The designed images were superimposed on the substrate. Then, the RTP film were fabricated by inkjet printing process from the premixed solution. Finally, these films were drying at $90^{\circ} \mathrm{C}$ for $2 \mathrm{~h}$ to finish the printing progress. (B) RTP photographs of eight patterns before and after continuous UV irradiation for $40 \mathrm{~s}$.
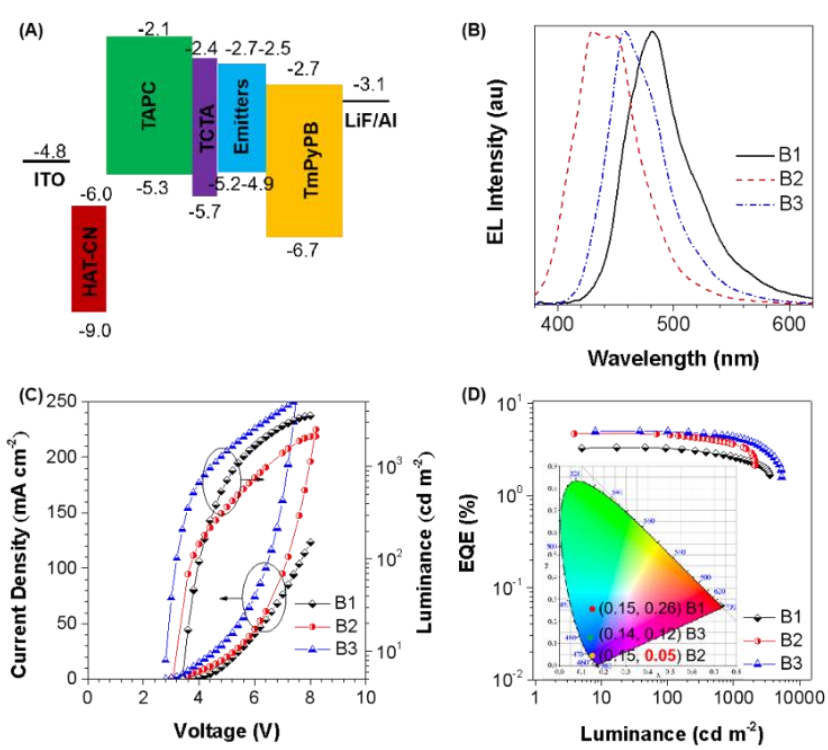

Figure 8. (A) Device structure. (B) Electroluminescence (EL) spectra of device B1-B3 at 3.0 V. (C) Current density -luminance-voltage (J-L-V) characteristics of devices B1-B3. (D) EQE versus luminance curves of the non-doped OLEDs based on these emitting materials. Device configuration: ITO/HAT-CN (5 nm)/TAPC (60 nm)/TCTA (5 nm)/EML (20 $\mathrm{nm}) / \mathrm{TmPyPB}(40 \mathrm{~nm}) / \mathrm{LiF}(1 \mathrm{~nm}) / \mathrm{Al}$. The B1 refers to the device with EML of 2PB-AC; B2: 2Me2PB-AC, and B3: 2T2PB-AC.

technology, thermal stability of 2PB-AC, 2Me2PB-AC and 2T2PB-AC were studied by differential scanning calorimetry (DSC) and thermogravimetric analysis (TGA) measurement. $T_{\mathrm{d}}$ (the temperatures for $5 \%$ weight loss) of $2 \mathrm{~PB}-\mathrm{AC}$, 2Me2PB-AC and 2T2PB-AC were recorded as high as 360, 339 , and $373{ }^{\circ} \mathrm{C}$, respectively, while their $T_{\mathrm{c}}$ (crystallization temperature) were deduced to be $194,185,220^{\circ} \mathrm{C}$, respectively (Figure S19). These data reveal that 2PB-AC, $2 \mathrm{Me} 2 \mathrm{~PB}-$ $\mathrm{AC}$ and $2 \mathrm{~T} 2 \mathrm{~PB}-\mathrm{AC}$ possess excellent thermal stability, which are suitable for OLED fabrication by the vacuum evaporation technique.

Moreover, the appropriate functional layers were selected for the construction of non-doped devices according to the acquired HOMO and LUMO energy levels of 2PB-AC, 2Me2PB-AC and 2T2PB-AC. Eventually, the device configuration of ITO/HAT-CN (5 nm)/TAPC (60 nm)/TCTA (5 $\mathrm{nm}) /$ EMLs $(20 \mathrm{~nm}) / \mathrm{TmPyPB}(40 \mathrm{~nm}) / \mathrm{LiF}(1 \mathrm{~nm}) / \mathrm{Al}$ was used (Figure $8 \mathrm{~A}$ ), where 1,4,5,8,9,11-hexaazatriphenylenehexacarbonitrile (HAT-CN), bis(4-( $N, N$-ditolyl-amino)-phenyl)cyclohexane (TAPC), tris(4-carbazoyl-9ylphenyl)amine (TCTA) and 1,3,5-tri(m-pyridin-3ylphenyl)benzene (TmPyPB) were selected as the hole injection, hole-transporting, exciton-blocking and electrontransporting layers, respectively. The device with EML of 2PB-AC, 2Me2PB-AC and 2T2PB-AC refers as B1, B2 and B3, respectively. The non-doped OLEDs emit in deep blue to blue regions with low operation voltage of 2.8-3.4 V (Figure 8B and 8C). Meanwhile, as shown in Figure S20-S22, their electroluminescence (EL) spectra are very stable with the increase of voltage, indicative of good color stability of the devices. Non-doped devices B1 and B3 exhibit maximum EQE value of $3.04 \%$ and $4.99 \%$ with CIE $(0.15,0.26)$ and $(0.14,0.12)$, respectively. Notably, the 2Me2PB-AC-based non-doped OLED achieves a maximum EQE of $4.75 \%$ with CIE coordinates of $(0.15,0.05)$, which is nearest to CIE coordinates $(x, y)=(0.15,0.046)$, defined by the UHDTV display standard blue color. The performances of non-doped devices B1-B3 were summarized in Table S3. Moreover, the non-doped devices present a very low efficiency roll-off due to the activation of the nonradiative transition and inhibition of the nonradiative transition channels in film state, further confirming their excellent device performances (Figures 8D, S23 and S24).

\section{Conclusions}

A practical molecular engineering strategy has been developed by combining planar terphenyl and D-A structures 
with different side chains, which achieves the luminogens with high emission in dilution solution and aggregate state. The terphenyl core and CT feature make molecular conformation more planar in the excited state, and thus the bright emission is observed in dilute solution. Moreover, the twisted TPA group jointed with cyano group can effectively restrict the molecular motions by forming the strong intermolecular interactions, which makes the compounds emit intensely in their powders. Furthermore, no obvious $\pi-\pi$ stacking of 2PB-AC confirmed by crystal structure is crucial for avoiding the non-radiative decay to enable strong emission in crystal state. Taking advantage of high emission in dilute state, these luminogens can act as the guests embedded into crystalline host and PMMA matrix to achieve efficient RTP as well as be used in advanced-encryption application. On the other hand, based on their superior thermal stability and high emission efficiency in solid states, excellent non-doped OLED with maximum EQE up to $4.75 \%$ and CIE $(0.15,0.05)$ was achieved. Our work provides a feasible strategy for the construction of two-phase highly emissive luminogens, which might broaden their practical applications.

\section{ASSOCIATED CONTENT}

Supporting Information. Supporting Information is available from ACS Publications website or from the author.

Materials and methods, synthetic procedures, characterization, computational details, photophysical properties, crystal packing, protocol of sample preparation, cyclic voltammograms curve, thermogravimetric and differential scanning calorimetry curve and device characterization including Figures S1-S36 and Tables S1-S3 (PDF).

[CCDC 2095218 contains the supplementary crystallographic data for this paper. These data can be obtained free of charge from The Cambridge Crystallographic Data Centre via www.ccdc.cam.ac.uk/data_request/cif.]

\section{Notes}

The authors declare no competing financial interest.

\section{ACKNOWLEDGMENT}

This work was financially supported by the National Natural Science Foundation of China (21788102), the Natural Science Foundation of Guangdong Province (2019B030301003 and 2016A030312002), and the Innovation and Technology Commission of Hong Kong (ITC-CNERC14S01).

\section{REFERENCES}

(1) Tang, C. W.; VanSlyke, S. A. Organic Electroluminescent Diodes. Appl. Phys. Lett. 1987, 51, 913-915.

(2) Poriel, C.; Rault Berthelot, J. Blue Single-Layer Organic LightEmitting Diodes Using Fluorescent Materials: a Molecular Design View Point. Adv. Funct. Mater. 2020, 30, 1910040.

(3) Shimizu, M.; Hiyama, T. Organic Fluorophores Exhibiting Highly Efficient Photoluminescence in the Solid State. Chem. Asian J. 2010, 5, 1516-1531.

(4) Liu, S.; Li, Y.; Kwok, R. T. K.; Lam, J. W. Y.; Tang, B. Z. Structural and Process Controls of AIEgens for NIR-II Theranostics. Chem. Sci. 2020, 12, 3427-3436.

(5) Wang, Y.; Han, X.; Xi, W.; Li, J.; Roe, A. W.; Lu, P.; Qian, J. Bright AIE Nanoparticles with F127 Encapsulation for Deep-Tissue
Three-Photon Intravital Brain Angiography. Adv. Healthc. Mater. 2017, 6, 1700685.

(6) Meher, N.; Iyer, P. K. Spontaneously Self-Assembled Naphthalimide Nanosheets: Aggregation-Induced Emission and Unveiling a-PET for Sensitive Detection of Organic Volatile Contaminants in Water. Angew. Chem. Int. Ed. 2018, 57, 8488-8492.

(7) Harmsen, S.; Bedics, M. A.; Wall, M. A.; Huang, R.; Detty, M. R.; Kircher, M. F. Rational Design of a Chalcogenopyrylium-Based Surface-Enhanced Resonance Raman Scattering Nanoprobe with Attomolar Sensitivity. Nat. Commun. 2015, 6, 1-9.

(8) Kwok, R. T.; Leung, C. W.; Lam, J. W.; Tang, B. Z. Biosensing by Luminogens with Aggregation Induced Emission Characteristics. Chem. Soc. Rev. 2015, 44, 4228-4238.

(9) Wu, H.; Zhao, Y. Colour-Tunable Ultra-Long Emission. Nat. Photonics 2019, 13, 373-375.

(10) Huang, B.; Chen, W.; Li, Z.; Zhang, J.; Zhao, W.; Feng, Y.; Tang, B. Z.; Lee, C. Manipulation of Molecular Aggregation States to Realize Polymorphism, AIE, MCL, and TADF in a Single Molecule. Angew. Chem. Int. Ed. 2018, 57, 12473-12477.

(11) Zhao, C.; Wakamiya, A.; Inukai, Y.; Yamaguchi, S. Highly Emissive Organic Solids Containing 2,5-Diboryl-1,4-phenylene Unit. J. Am. Chem. Soc. 2006, 128, 15934-15935.

(12) Huang, Y.; Xing, J.; Gong, Q.; Chen, L.-C.; Liu, G.; Yao, C.; Wang, Z.; Zhang, H.-L.; Chen, Z.; Zhang, Q. Reducing Aggregation Caused Quenching Effect Through Co-Assembly of PAH Chromophores and Molecular Barriers. Nat. Commun. 2019, 10, 1-9.

(13) Mei, J.; Leung, N. L. C.; Kwok, R. T. K.; Lam, J. W. Y.; Tang, B. Z. Aggregation-Induced Emission: Together We Shine, United We Soar! Chem. Rev. 2015, 115, 11718-11940.

(14) Sasaki, S.; Suzuki, S.; Sameera, W. M. C.; Igawa, K.; Morokuma, K.; Konishi, G. Highly Twisted N, N -Dialkylamines as a Design Strategy to Tune Simple Aromatic Hydrocarbons as Steric EnvironmentSensitive Fluorophores. J. Am. Chem. Soc. 2016, 138, 8194-8206.

(15) Yang, J.; Chi, Z.; Zhu, W.; Tang, B. Z.; Li, Z. Aggregation-Induced Emission: a Coming-of-Age Ceremony at the Age of Eighteen. Sci. China Chem. 2019, 62, 1090-1098.

(16) Zhao, Z.; Zhang, H.; Lam, J. W. Y.; Tang, B. Z. Aggregation-Induced Emission: New Vistas at the Aggregate Level. Angew. Chem. Int. Ed. 2020, 59, 9972-9993.

(17) Zhang, H.; Zhao, Z.; Turley, A. T.; Wang, L.; McGonigal, P. R.; Tu, Y.; Li, Y.; Wang, Z.; Kwok, R. T. K.; Lam, J. W. Y.; Tang, B. Z. Aggregate Science: From Structures to Properties. Adv. Mater. 2020, 32, 2001457.

(18) Chen, Y.; Lam, J. W. Y.; Kwok, R. T. K.; Liu, B.; Tang, B. Z. Aggregation-Induced Emission: Fundamental Understanding and Future Developments. Mater. Horiz. 2019, 6, 428-433.

(19) Cao, H.; Yang, Y.; Li, J. AIEgen-Lipid Structures: Assembly and Biological Applications. Aggregate 2020, 1, 69-79.

(20) Nie, H.; Hu, K.; Cai, Y.; Peng, Q.; Zhao, Z.; Hu, R.; Chen, J.; Su, S.; Qin, A.; Tang, B. Z. Tetraphenylfuran: Aggregation-Induced Emission or Aggregation-Caused Quenching? Mater. Chem. Front. 2017, 1, 1125-1129.

(21) Yan, X.; Cook, T. R.; Wang, P.; Huang, F.; Stang, P. J. Highly emissive platinum(II) metallacages. Nat. Chem. 2015, 7, 342-348.

(22) Zheng, X.; Zhu, W.; Zhang, C.; Zhang, Y.; Zhong, C.; Li, H.; Xie, G.; Wang, X.; Yang, C. Self-Assembly of a Highly Emissive Pure Organic Imine-Based Stack for Electroluminescence and Cell Imaging. J. Am. Chem. Soc. 2019, 141, 4704-4710.

(23) Chen, G.; Li, W.; Zhou, T.; Peng, Q.; Zhai, D.; Li, H.; Yuan, W. Z.; Zhang, Y.; Tang, B. Z. Conjugation-Induced Rigidity in Twisting Molecules: Filling the Gap Between Aggregation-Caused Quenching and Aggregation-Induced Emission. Adv. Mater. 2015, 27, 44964501.

(24) Wu, H.; Chen, Z.; Chi, W.; Bindra, A. K.; Gu, L.; Qian, C.; Wu, B.; Yue, B.; Liu, G.; Yang, G.; Zhu L.; Zhao Y. Structural Engineering of Luminogens with High Emission Efficiency both in Solution and in the Solid State. Angew. Chem. Int. Ed. 2019, 58, 11419-11423.

(25) Li, X.; Baryshnikov, G.; Ding, L.; Bao, X.; Li, X.; Lu, J.; Liu, M.; Shen, S.; Luo, M.; Zhang, M.; Ågren H.; Wang X.; Zhu L. Dual-Phase 
Thermally Activated Delayed Fluorescence Luminogens: A Material for Time-Resolved Imaging Independent of Probe Pretreatment and Probe Concentration. Angew. Chem. Int. Ed. 2020, 59, 7548-7554.

(26) Chen, W.; Chen, C.; Zhang, Z.; Chen, Y.; Chao, W.; Su, J.; Tian, H.; Chou, P. Snapshotting the Excited-State Planarization of Chemically Locked N,N'-Disubstituted Dihydrodibenzo[a ,c]phenazines. J. Am. Chem. Soc. 2017, 139, 1636-1644.

(27) Zhang, Z.; Chen, C.; Chen, Y.; Wei, Y.; Su, J.; Tian, H.; Chou, P. Tuning the Conformation and Color of Conjugated Polyheterocyclic Skeletons by Installingortho -Methyl Groups. Angew. Chem. Int. Ed. 2018, 57, 9880-9884.

(28) Li, L.; Chen, M.; Zhang, H.; Nie, H.; Sun, J.; Qin, A.; Tang, B. Z. Influence of the Number and Substitution Position of Phenyl Groups on the Aggregation-Enhanced Emission of Benzene-Cored Luminogens. Chem. Commun. 2015, 51, 4830-4833.

(29) Yuan, W. Z.; Lu, P.; Chen, S.; Lam, J. W. Y.; Wang, Z.; Liu, Y.; Kwok, H. S.; Ma, Y.; Tang, B. Z. Changing the Behavior of Chromophores from Aggregation-Caused Quenching to Aggregation-Induced Emission: Development of Highly Efficient Light Emitters in the Solid State. Adv. Mater. 2010, 22, 2159-2163.

(30) Reva, I.; Lapinski, L.; Chattopadhyay, N.; Fausto, R. Vibrational Spectrum and Molecular Structure of Triphenylamine Monomer: A Combined Matrix-Isolation FTIR and Theoretical Study. Phys. Chem. Chem. Phys. 2003, 5, 3844-3850.

(31) Malagoli, M.; Brédas, J. L. Density Functional Theory Study of the Geometric Structure and Energetics of Triphenylamine-Based Hole-Transporting Molecules. Chem. Phys. Lett. 2000, 327, 13-17. (32) Han, P.; Xu, Z.; Lin, C.; Ma, D.; Qin, A.; Tang, B. Z. Tetraphenylbenzene-Based AIEgens: Horizontally Oriented Emitters for Highly Efficient Non-Doped Deep Blue OLEDs and Hosts for HighPerformance Hybrid WOLEDs. J. Mater. Chem. C 2020, 8, 70127018.

(33) Li, L.; Nie, H.; Chen, M.; Sun, J.; Qin, A.; Tang, B. Z. AggregationEnhanced Emission Active Tetraphenylbenzene-Cored Efficient Blue Light Emitter. Faraday Discuss. 2017, 196, 245-253.

(34) Lee, J.; Chen, C.; Lee, P.; Lin, H.; Leung, M.; Chiu, T.; Lin, C. Blue Organic Light-Emitting Diodes: Current Status, Challenges, and Future Outlook. J. Mater. Chem. C 2019, 7, 5874-5888.

(35) Han, P.; Lin, C.; Ma, D.; Qin, A.; Tang, B. Z. Violet-Blue Emitters Featuring Aggregation-Enhanced Emission Characteristics for Nondoped OLEDs with CIEy Smaller than 0.046. ACS Appl. Mater. Inter. 2020, 12, 46366-46372.

(36) Pan, L.; Cai, Y.; Wu, H.; Zhou, F.; Qin, A.; Wang, Z.; Tang, B. Z. Tetraphenylpyrazine-Based Luminogens with Full-Colour Emission. Mater. Chem. Front. 2018, 2, 1310-1316.

(37) Hu, R.; Lager, E.; Aguilar-Aguilar, A.; Liu, J.; Lam, J. W. Y.; Sung, H. H. Y.; Williams, I. D.; Zhong, Y.; Wong, K. S.; Peña-Cabrera, E.; Tang, B. Z. Twisted Intramolecular Charge Transfer and Aggregation-Induced Emission of BODIPY Derivatives. J. Phys. Chem. C 2009, 113, 15845-15853.

(38) Tu, Y.; Yu, Y.; Xiao, D.; Liu, J.; Zhao, Z.; Liu, Z.; Lam, J. W. Y.; Tang, B. Z. An Intelligent AIEgen with Nonmonotonic Multirespones to Multistimuli. Adv. Sci. 2020, 7, 2001845.

(39) Zhang, H.; Zhang, B.; Zhang, Y.; Xu, Z.; Wu, H.; Yin, P. A.; Wang, Z.; Zhao, Z.; Ma, D.; Tang, B. Z. A Multifunctional Blue-Emitting Material Designed via Tuning Distribution of Hybridized Excited-State for High-Performance Blue and Host-Sensitized OLEDs. Adv. Funct. Mater. 2020, 30, 2002323.

(40) Lum, K.; Zielinski, S. M.; Abelt, C. J. Dansyl Emits from a PICT Excited State. J. Phys. Chem. A 2021, 125, 1229-1233.

(41) Park, I. S.; Matsuo, K.; Aizawa, N.; Yasuda, T. High-Performance Dibenzoheteraborin-Based Thermally Activated Delayed Fluorescence Emitters: Molecular Architectonics for Concurrently Achieving Narrowband Emission and Efficient Triplet-Singlet Spin Conversion. Adv. Funct. Mater. 2018, 28, 1802031.

(42) Chen, F.; Zhao, L.; Wang, X.; Yang, Q.; Li, W.; Tian, H.; Shao, S.; Wang, L.; Jing, X.; Wang, F. Novel Boron- and Sulfur-Doped Polycy- clic Aromatic Hydrocarbon as Multiple Resonance Emitter for Ultrapure Blue Thermally Activated Delayed Fluorescence Polymers. Sci. China Chem. 2021, 64, 547-551.

(43) Yin, H.; Wang, X.; Yin, X. Rotation Restricted Emission and Antenna Effect in Single Metal-Organic Frameworks. J. Am. Chem. Soc. 2019, 141, 15166-15173.

(44) Lin, Z.; Kabe, R.; Nishimura, N.; Jinnai, K.; Adachi, C. Organic Long-Persistent Luminescence from a Flexible and Transparent Doped Polymer. Adv. Mater. 2018, 30, 1803713.

(45) Lei, Y.; Dai, W.; Tian, Y.; Yang, J.; Li, P.; Shi, J.; Tong, B.; Cai, Z.; Dong, Y. Revealing Insight into Long-Lived Room-Temperature Phosphorescence of Host-Guest Systems. J Phys Chem. Lett. 2019, 10, 6019-6025.

(46) Li, D.; Lu, F.; Wang, J.; Hu, W.; Cao, X.; Ma, X.; Tian, H. Amorphous Metal-Free Room-Temperature Phosphorescent Small Molecules with Multicolor Photoluminescence via a Host-Guest and Dual-Emission Strategy. J. Am. Chem. Soc. 2018, 140, 1916-1923.

(47) Qiu, W.; Cai, X.; Li, M.; Chen, Z.; Wang, L.; Xie, W.; Liu, K.; Liu, M.; Su, S. Achieving Purely Organic Room-Temperature Phosphorescence Mediated by a Host-Guest Charge Transfer State. J. Phys. Chem. Lett. 2021, 12, 4600-4608.

(48) Xie, Z.; Zhang, X.; Wang, H.; Huang, C.; Sun, H.; Dong, M.; Ji, L.; An, Z.; Yu, T.; Huang, W. Wide-Range Lifetime-Tunable and Responsive Ultralong Organic Phosphorescent Multi-Host/Guest System. Nat. Commun. 2021, 12, 1-8.

(49) Ning, Y.; Yang, J.; Si, H.; Wu, H.; Zheng, X.; Qin, A.; Tang, B. Z. Ultralong Organic Room-Temperature Phosphorescence of Electron-Donating and Commercially Available Host and Guest Molecules Through Efficient Förster Resonance Energy Transfer. Sci. China Chem. 2021, 64, 739-744.

(50) Tang, S.; Li, W.; Shen, F.; Liu, D.; Yang, B.; Ma, Y. Highly Efficient Deep-Blue Electroluminescence Based on the TriphenylamineCored and Peripheral Blue Emitters with Segregative HOMO-LUMO Characteristics. J. Mater. Chem. 2012, 22, 4401-4408.

(51) Wang, Y.; Yang, J.; Fang, M.; Yu, Y.; Zou, B.; Wang, L.; Tian, Y.; Cheng, J.; Tang, B. Z.; Li, Z. Förster Resonance Energy Transfer: An Efficient Way to Develop Stimulus-Responsive Room-Temperature Phosphorescence Materials and Their Applications. Matter 2020, 3, 449-463.

(52) Sun, Y.; Giebink, N. C.; Kanno, H.; Ma, B.; Thompson, M. E.; Forrest, S. R. Management of Singlet and Triplet Excitons for Efficient White Organic Light-Emitting Devices. Nature 2006, 440, 908-912. (53) Sun, L.; Zhu, W.; Wang, W.; Yang, F.; Zhang, C.; Wang, S.; Zhang, X.; Li, R.; Dong, H.; Hu, W. Intermolecular Charge-Transfer Interactions Facilitate Two-Photon Absorption in Styrylpyridine-Tetracyanobenzene Cocrystals. Angew. Chem. Int. Ed. 2017, 56, 7831-7835. (54) Ma, L.; Sun, S.; Ding, B.; Ma, X.; Tian, H. Highly Efficient RoomTemperature Phosphorescence Based on Single-Benzene Structure Molecules and Photoactivated Luminescence with Afterglow. Adv. Funct. Mater. 2021, 31, 2010659.

(55) Gu, L.; Shi, H.; Gu, M.; Ling, K.; Ma, H.; Cai, S.; Song, L.; Ma, C.; Li, H.; Xing, G.; Hang X.; Li J.; Gao Y.; Yao Wei.; Shuai Z.; An Z.; Liu X.; Huang W. Dynamic Ultralong Organic Phosphorescence by Photoactivation. Angew. Chem. Int. Ed. 2018, 57, 8425-8431.

(56) Garain, S.; Kuila, S.; Garain, B. C.; Kataria, M.; Borah, A.; Pati, S. K.; George, S. J. Arylene Diimide Phosphors: Aggregation Modulated Twin Room Temperature Phosphorescence from Pyromellitic Diimides. Angew. Chem. Int. Ed. 2021, 133, 12431-12435.

(57) Ieuji, R.; Goushi, K.; Adachi, C. Triplet-Triplet Upconversion Enhanced by Spin-Orbit Coupling in Organic Light-Emitting Diodes. Nat. Commun. 2019, 10, 1-10. 


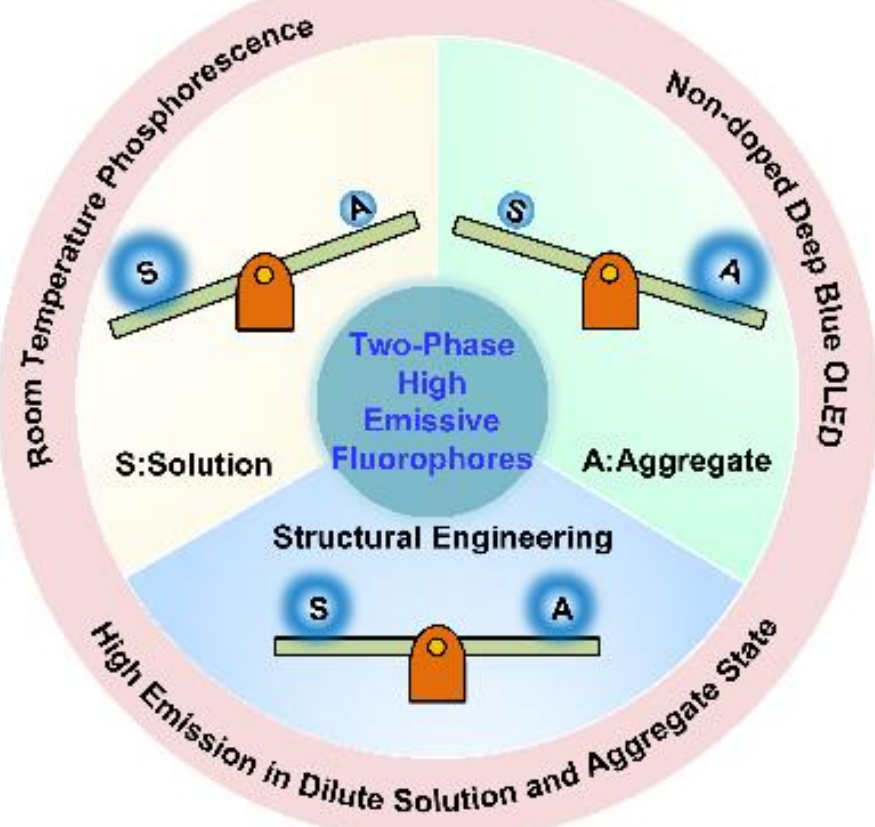

Table of Contents 